\title{
Involvement of the nervous system following experimental infection with Pasteurella multocida B:2 in buffalo (Bubalus bubalis): a clinicopathological study
}

\begin{abstract}
Haemorrhagic septicaemia (HS) is an acute, fatal, septicaemic disease of cattle and buffaloes caused by one of two specific serotypes of Pasteurella multocida B:2 and E:2 in Asian and African, respectively. It is well known that HS affect mainly the respiratory and digestive tracts. However, involvement of the nervous system in pathogenesis of HS has been reported in previous studies without details. In this study, nine buffalo calves of 8 months old were distributed into three groups. Animals of Group 1 and 2 were inoculated orally and subcutaneously with $10 \mathrm{ml}$ of $1 \times 1012 \mathrm{cfu} / \mathrm{ml}$ of P. multocida B:2, respectively, while animals of Group 3 were inoculated orally with $10 \mathrm{ml}$ of phosphate buffer saline as a control. All calves in Group 1 and Group 3 were euthanised after 504 h (21 day) post-infection, while calves in Group 2 had to euthanise after $12 \mathrm{~h}$ post-infection as they develop sever clinical signs of HS. Significant differences were found in Group 2 in the mean scores of clinical signs, gross and histopathological changes which mainly affect different anatomic regions of the nervous system. In addition, successful bacterial isolation of P. multocida B:2 were obtained from different sites of the nervous system. On the other hand, less sever, clinical, gross and histopathological changes were found in Group 1. These results provide for the first time strong evidence of involving of the nervous system in pathogenesis of HS, especially in the peracute stage of the disease.
\end{abstract}

Keyword: Haemorrhagic septicaemia; Buffaloes; Pasteurella multocida B:2; Nervous system; Clinicopathological 